\title{
R16 - DEVELOPMENT OF A REVERSE TRANSCRIPTION QUANTITATIVE REAL-TIME PCR-BASED SYSTEM FOR RAPID DETECTION AND QUANTIFICATION OF HEPATITIS DELTA VIRUS
}

Luan Felipo Botelho de Souza ${ }^{1} 2$ 3*, Alcione de Oliveira dos Santos ${ }^{1} 23$, Lourdes Maria

Borzacov ${ }^{2}$,Eduardo Resende Honda ${ }^{2}$, Juan Miguel Villalobos-Salcedo ${ }^{1} 23$ and Deusilene $\underline{\text { Souza Vieira }{ }^{1} 23}$

1. Fundação Oswaldo Cruz Rondônia, Brazil-FIOCRUZ-RO; 2. Centro de Pesquisa em Medicina Tropical de Rondônia, Brazil - CEPEM and 3. Universidade Federal de Rondonia, Brazil - UNIR

Objectives: This study we developed an in-house assay capable of detecting and quantifying hepatitis delta virus using serum samples based on a reverse transcription quantitative Real-Time PCR (RT-qPCR HDV).

Methods: The study included 100 serum samples from patients infected with HBV/HDV. To test the specificity, a control group of 30 blood donors was included. For HDV RNA extraction was performed with the QIAamp Viral RNA Mini Kit (Qiagen, Germany). HDV RNA was converted in cDNA using 200 units of M-MLV enzyme (Sigma Aldrich ${ }^{\circledR}$, Saint Louis, USA). The primers and hydrolysis probe (TaqMan Probes) were designed based on known and complete HDV sequences that are deposited in GenBank. For determining the viral load were produced in-house two calibrators standard HDV, a cDNA cloned into the plasmid and another transcribed RNA. For the validation this assay were used 140 clinical samples of serum, constituted of 100 samples from patients AntiHDV and HBsAg positive by ELISA, 30 samples of blood donors, 5 samples with monoinfection $\mathrm{HBV}$ and 5 with monoinfection $\mathrm{HCV}$.

Results: The limits of detection were $1.3 \times 10^{2}$ and $8.4 \times 101$ copies $/ \mathrm{mL}$ for the calibrator standard HDV cDNA and RNA. From 100 serum samples of HBsAg- and anti-HDV positive patients who were tested by performing HDV-qPCR, 54\% were positive with a viral load greater than or equal to the detection limit and $46 \%$ were considered to be negative because they were below the detection limit of the assay as seen by the standard 
calibrator HDV cDNA. All 30 samples from the blood donors were negative. The linearity of HDV-qPCR was evaluated by constructing a linear regression curve for each run of serial dilutions of 6- $\log _{10}$ calibrator standard HDV cDNA and RNA, whereby we found a strong correlation between the dilutions (correlation coefficient $\mathrm{R}^{2}=0.97$ and 0.99 ) for both standards. The amplification efficiency was calculated from the slope of -3325 (99.8\%) and -2.92 (119\%, unsatisfying) for calibrator standard HDV cDNA and RNA, respectively. Repeatability was measured by determining the standard deviation (SD) of the intra-assay where the SD varied from 0.27 to 0.71 and from 0.02 to 0.35 for calibrator standard HDV cDNA and RNA. The reproducibility of the HDV-qPCR assay was confirmed by the inter-assay where the HDV RNA was quantified in serum samples of 6 $\times 3$ runs on consecutive days.

The HDV-qPCR assay is highly capable of determining the viral load of serum samples with low variability between runs.

Conclusion: This study represents the first HDV RT-qPCR assay developed in the Western Amazon region and offers great potential for new clinical efficacy studies of therapeutic antiviral used in patients with hepatitis delta in this region. 